\title{
Atypical Kawasaki Disease in Adolescent Male with Liver Involvement Following Human Coronavirus OC43 Infection
}

\author{
Fatima Bohlok ${ }^{1}$, Zahraa Abou Hamdan ${ }^{1}$, Naima Rachini ${ }^{1}$, Sandy \\ Rabah $^{2}$, Zahraa Slim ${ }^{2}$, and Lina Mortada ${ }^{3 *}$ \\ ${ }^{1}$ Department of Pediatrics, Pediatric Resident PGY4, Lebanese University, Faculty of \\ Medical Sciences, Lebanon \\ ${ }^{2}$ Department of Pediatrics, Pediatric Resident PGY3, Lebanese University, Faculty of \\ Medical Sciences, Lebanon \\ ${ }^{3}$ Pediatrician, Department of Pediatrics, Lebanese University, Faculty of Medical \\ Sciences, Lebanon \\ *Corresponding Author: Lina Mortada, Pediatrician, Department of Pediatrics, \\ Lebanese University, Faculty of Medical Sciences, Lebanon.
}

DOI: 10.31080/ASPE.2020.03.0225

\author{
Received: January 21, 2020; \\ Published: February 17, 2020 \\ (c) All rights are reserved by Lina Mortada., \\ et al.
}

\begin{abstract}
Kawasaki disease is an acute febrile illness of early childhood; less than one per cent of cases are older than ten years. We report here a 13 and half years old male patient presenting with high grade fever, asthenia, myalgia, arthralgia and liver involvement followed by non-exudative conjunctivitis, skin rash on extremities plus desquamation; viral PCR panel was positive for human coronavirus HCOV-OC 43; Blood test showed elevated inflammatory markers, cholestasis and increased platelet count. After elimination of viral, bacterial and autoimmune causes we started IVIG for incomplete Kawasaki disease with good response in 24 hrs.

Kawasaki disease is uncommon in adolescent patients, so diagnosis could be delayed increasing the risk of coronary artery anevrysm; also the cause is unknown, it may be caused by some viral illness and in our case it turn to be the human coronavirus HCOVOC 43; liver involvement can be present in Kawasaki disease and it's due to hepatobiliary dysfunction.

Adolescent with Kawasaki disease may have atypical presentation, the diagnosis can be difficult; we suggest to have a high index of suspicion in evaluating adolescent patients with high grade fever of unknown origin regarding the possibility of having atypical Kawasaki and the possible coronary artery disease.

Keywords: Kawasaki Disease; Human Coronavirus OC43; Liver Involvement; Adolescent Patient; Vasculitis
\end{abstract}

\section{Abbreviations}

ANA: Anti-Nuclear Antibody; ANCA: Anti-Nuclear Cytoplasmic Antibody; CMV: Cytomegalovirus; CRP: C-Reactive Protein; EBV: Epstein-Barr Virus; GGT: Gamma-Glutamyl Transpeptidase; HCovs: Human Coronavirus; HCov 43: Human Coronavirus oc 43; HIV: Human Immunodeficiency Virus; IVIG: Intra-Venous Immune Globulin; PCR: polymerase Chain Reaction; SARS: Severe Acute Respiratory Syndrome.

\section{Introduction}

Kawasaki disease is an acute febrile illness of early childhood (less than five years); characterized by vasculitis of the mediumsized arteries [3].

Children older than ten years with Kawasaki disease account for less than one percent of all the cases [1,2]. The diagnosis of Kawasaki disease is based entirely on clinical features. For classic
Kawasaki disease, individuals must have a fever for $>5$ days and either meet at least 4 of the 5 following criteria:

- Bilateral conjunctivitis.

- Eryhtema of the mouth or pharynx, strawberry tongue, or stomatitis.

- Polymorphous rash.

- Eryhtema or edema of the hands or feet.

- Non suppurative cervical lymphadenopathy. Or meet at least 3 of these criteria and have evidence of coronary artery abnormality [4].

Incomplete or atypical Kawasaki disease, in which these criteria are not met, can occur and can also result in aneurysms of the coronary arteries [5]. The etiology of Kawasaki disease is unknown. Laboratory findings are nonspecific, and there are no diagnostic tests for Kawasaki disease. 
There is an evidence which suggests that Kawasaki disease may be triggered by a response to an infectious agent [6]. The widespread immunity to a common infectious agent may explain the rarity of Kawasaki disease in adults. A history of an antecedent respiratory illness before the onset of Kawasaki disease has been reported [7]. Although many infectious agents have been proposed as the cause of Kawasaki disease, none have been consistently associated with the disease. Human coronaviruses (HCoVs) have attracted renewed interest recently, in part because of the emergence of a novel $\mathrm{HCoV}$ associated with severe acute respiratory syndrome (SARS) $[8,9]$.

We report an adolescent male with Kawasaki disease presenting with liver involvement and infected by Human Coronavirus oc43.

\section{Case Report}

A 13 and half years old Lebanese male patient, who is an smoker (4 packs/year), presented to the emergency department with a two days history of high grade fever with chills, cough, and severe fatigue. Chest $-x$ ray showed bilateral infiltrates; laboratory investigations showed neutrophilic leukocytosis $\left(19.3 \times 10^{\wedge} 9 / 1\right)$, normal platelets $\left(400 \times 10^{\wedge} 3 / \mathrm{l}\right)$, elevated C-reactive protein $(59 \mathrm{mg} / \mathrm{l})$ and erythrocyte sedimentation rate of $(60 \mathrm{~mm} / \mathrm{h})$. Diagnosed as bronchopneumonia, started on IV ceftriaxone. 5 days prior to presentation, he had 3 days history of fever and cough, treated with azithromycin with improvement and subsidence of fever.

His medical history was unremarkable and he denied drug intake. Two days later he got mild jaundice with bilateral icteric sclera, also bilateral rhonchi. Asthenia and myalgia were noted.

Liver function tests showed elevated alanine transaminase (ALT $=613 \mathrm{U} / \mathrm{L})$, elevated gamma-glutamyl transferase $(\mathrm{GGT}=268 \mathrm{U} / \mathrm{L})$, elevated total/direct bilirubin (5.1/3.4). fever was persistent. Serologic assays for Human Immunodeficiency Virus (HIV), Influenza test, Hepatotropic virus EBV and CMV were negative. Wright and Widal tests also were negative.

On the third day of hospitalization, patient complained from abdominal pain. Repeated laboratory tests showed persistent elevation in C-Reactive Protein and Erythrocyte Sedimentation Rate, levels of D-Dimer and fibrinogen were significantly elevated (1300 microg/l; $1754 \mathrm{mg} /$ dl respectively), lactate dehydrogenase was normal (130); ferritin level was high (626). Amylase and lipase were slightly elevated (160, 86 IU/L respectively). Abdominal ultrasound showed hepatomegaly and alithiasic cholestasis.

Respiratory Viral panel PCR was positive for Human Coronavirus OC43. Neuro 9 was negative. Panculture was negative. Periph- eral smear showed leukocytosis with toxic granulations in neutrophils.

After 4 days of hospitalization, patient still had high grade fever associated with asthenia, arthralgia and severe abdominal pain. So repeated test showed persistence of leukocytosis $\left(20.4 \times 10^{\wedge} 9 / \mathrm{l}\right)$; thrombocytosis $\left(640 \times 10^{\wedge} 3\right)$; elevated $\mathrm{C}$ - reactive protein $(408)$ and elevated erythrocyte sedimentation rate (130). Bilirubin, GGT and triglycerides still increasing. C3, C4, ANA, anti-DNA, ANCA were negative. CT scan abdomino-pelvic showed hepatomegaly and alithiasic cholestasis. So he was shifted to meropenem, vancomycin and fluconazole. Bone marrow aspirate, biopsy and culture for salmonella and brucella, all were negative.

On the 5th day of hospitalization, patient still febrile, he started to have bilateral non exudative conjunctivitis and skin rash on hands and feet with desquamation on hands and genital area. Repeated labs showed persistence of increased platelets count, Creactive protein, and erythrocyte sedimentation rate.

The patient was considered to have incomplete Kawasaki disease and he was given single dose of IVIG $(2 \mathrm{~g} / \mathrm{kg}$ ) over 12 hours, and anti-inflammatory dose of acetyl salicylic acid ( $4 \mathrm{~g} /$ day divided into 4 doses). Cardiac ultrasound showed no abnormality.

Twenty four hours after IVIG, the patient's fever returned to normal. Inflammatory blood tests were decreasing, Patient was discharged home. After one week, repeated cardiac ultrasound was normal; sedimentation rate and CRP value decreased. Aspirin was reduced to only one dose of $3 \mathrm{mg} / \mathrm{kg}$.

\section{Discussion}

Kawasaki disease is uncommon in adolescent age group. Because of atypical presentation in older children, the diagnosis of Kawasaki disease is difficult and delayed [10].

The cause of Kawasaki disease has been elusive. Epidemiological data suggest that Kawasaki disease may be caused by a common infectious agent. Several pathogens such as retrovirus, EBV, parvovirus B19, and chlamydia have been suggested by different studies as possibly important in the pathogenesis of Kawasaki disease. In this patient, there was an association between Human coronavirus (HCoV-OC43) infection and Kawasaki disease.

Also, Kawasaki disease is associated with hydrops gallbladder, intrahepatic bile duct damage and hepatobiliary dysfunction. Mild impairment of liver function test is a common finding in Kawasaki disease. However, jaundice or elevated bilirubin is uncommon. Jaundice may be caused by the intrahepatic cause due to hepatobiliary dysfunction. The likely cause of hepatic involvement in our 
patient is due to hepatocellular injury associated with Kawasaki disease. Our patient presented with respiratory infection caused by HCoV-OC43 and liver involvement. This association was reported in the literature, with hepatic impairment in patient with SARS (severe acute respiratory syndrome) which is due to SARS -associated coronavirus infection of the liver [11]. The possibility of viral etiology is established. However; this rare presentation should be alerted as prompt treatment with IVIG will help in controlling the systemic inflammation as well as decreasing the chance of development of coronary aneurysm. This child represents an atypical case by age of presentation.

The diagnosis can often be delayed as pediatricians are less likely to consider Kawasaki disease at extreme age with unusual presentation. Hence pediatricians should be alert of the possibility of incomplete Kawasaki disease in adolescence with bizarre presentation, as in this case, and seek a second opinion. Follow up echocardiogram is recommended in case of doubt.

\section{Conclusion}

Kawasaki disease presenting with liver involvement induced by Human Coronavirus oc43 is a rare presentation. Adolescents with Kawasaki disease may have atypical presentation. They are at high risk of coronary aneurysm formation. The diagnosis can be late with a higher complication rate of coronary aneurysm. Clinician should have a high index of suspicion in evaluating patients presenting with fever of unknown origin in children beyond the typical age of Kawasaki disease.

\section{Bibliography}

1. Stockheim JA., et al. "Kawasaki disease in older children and adolescents". Journal of Pediatrics 137 (2000): 250-252.

2. Momenah T., et al. "Kawasaki disease in the older child". Pediatrics 102 (1998): e7.

3. Son MBF and Newburger JW. "Kawasaki disease”. Pediatric Review 39.2 (2018): 78-90.

4. American Academy of Pediatrics. Red book: report of the Committee on Infectious Diseases, 25th ed., Elk Grove Village, IL American Academy of Pediatrics (2000).

5. Rosenfeld EA., et al. "Kawasaki disease in infants less than one year of age". Journal of Pediatrics 126 (1995): 524-529.

6. Yanagawa H., et al. "Nationwide epidemic of Kawasaki disease in Japan during winter of 1985-86". Lancet 2 (1986): 11381139.
7. Bell DM., et al. "Kawasaki syndrome: description of two outbreaks in the United States". The New England Journal of Medicine 304 (1981): 568-575.

8. Ksiazek TG., et al. "A novel coronavirus associated with severe acute respiratory syndrome". The New England Journal of Medicine 348 (2003): 1953-1966.

9. Drosten C., et al. "Identification of a novel coronavirus in patients with severe acute respiratory syndrome". The New England Journal of Medicine 348 (2003): 1967-1976.

10. Han RK., et al. "Management and outcome of persistent or recurrent fever after initial intravenous gamma globulin therapy in acute Kawasaki disease". Archives of Pediatrics and Adolescent Medicine 154 (2000): 694-699.

11. Chau TN., et al. "SARS-associated viral hepatitis caused by a novel coronavirus: report of three cases". Hepatology 39.2 (2004): 302-310.)

\section{Assets from publication with us}

- Prompt Acknowledgement after receiving the article

- Thorough Double blinded peer review

- Rapid Publication

- Issue of Publication Certificate

- High visibility of your Published work

Website: www.actascientific.com/

Submit Article: www.actascientific.com/submission.php Email us: editor@actascientific.com

Contact us: +919182824667 